\title{
Correction to: Classification of non-local rings with genus two zero-divisor graphs
}

\author{
T. Asir ${ }^{1}$ (1) $\cdot$ K. Mano ${ }^{1} \cdot$ T. Tamizh Chelvam ${ }^{2}$
}

Published online: 30 January 2021

○) Springer-Verlag GmbH Germany, part of Springer Nature 2021

\section{Correction to: \\ Soft Computing (2020) 24:237-245 https://doi.org/10.1007/s00500-019-04345-0}

The purpose of this note is to correct some errors in the article [1]. The notation here will follow that of the original article.

It was argued in [1] that the graph $\Gamma\left(\mathbb{Z}_{2} \times \mathbb{Z}_{2} \times \mathbb{Z}_{6}\right)$ or $\Gamma\left(\mathbb{Z}_{2} \times \mathbb{Z}_{2} \times \mathbb{F}_{8}\right)$ is (isomorphic to) a subgraph of $\Gamma\left(\mathbb{Z}_{2} \times \mathbb{Z}_{2} \times \mathbb{Z}_{8}\right)$ and $\Gamma\left(\mathbb{Z}_{2} \times \mathbb{Z}_{2} \times \mathbb{Z}_{2}[x] /\left(x^{3}\right)\right)$ is isomorphic to $\Gamma\left(\mathbb{Z}_{2} \times \mathbb{Z}_{2} \times \mathbb{Z}_{8}\right)$. Using these facts it was proved that $\gamma\left(\Gamma\left(\mathbb{Z}_{2} \times \mathbb{Z}_{2} \times \mathbb{Z}_{6}\right)\right)=\gamma\left(\Gamma\left(\mathbb{Z}_{2} \times \mathbb{Z}_{2} \times \mathbb{F}_{8}\right)\right)=$ $\gamma\left(\Gamma\left(\mathbb{Z}_{2} \times \mathbb{Z}_{2} \times \mathbb{Z}_{2}[x] /\left(x^{3}\right)\right)\right)=2$. Actually, the degree three vertices namely $(0,1,6),(1,0,6)$ and $(1,1,4)$ of the graph $\Gamma\left(\mathbb{Z}_{2} \times \mathbb{Z}_{2} \times \mathbb{Z}_{8}\right)$ have not been included in the $S_{2}$ embedding of $\Gamma\left(\mathbb{Z}_{2} \times \mathbb{Z}_{2} \times \mathbb{Z}_{8}\right)$ provided in Fig. 5 of [1]. This mistake causes a change in the value the genus of $\Gamma\left(\mathbb{Z}_{2} \times \mathbb{Z}_{2} \times \mathbb{Z}_{8}\right)$. So in this note, we find the exact genus value of the following four rings:

$\mathbb{Z}_{2} \times \mathbb{Z}_{2} \times \mathbb{F}_{8}, \mathbb{Z}_{2} \times \mathbb{Z}_{2} \times \mathbb{Z}_{6}, \mathbb{Z}_{2} \times \mathbb{Z}_{2} \times \mathbb{Z}_{8}, \mathbb{Z}_{2} \times \mathbb{Z}_{2} \times \mathbb{Z}_{2}[x] /\left(x^{3}\right)$.

First of all, a $S_{2}$-embedding of $\Gamma\left(\mathbb{Z}_{2} \times \mathbb{Z}_{2} \times \mathbb{F}_{8}\right)$ is given in Fig. 1 so that $\gamma\left(\Gamma\left(\mathbb{Z}_{2} \times \mathbb{Z}_{2} \times \mathbb{F}_{8}\right)\right)=2$.

Also, by Fig. 2 , we get $\gamma\left(\Gamma\left(\mathbb{Z}_{2} \times \mathbb{Z}_{2} \times \mathbb{Z}_{6}\right)\right)=2$ and so $\gamma\left(\Gamma\left(\mathbb{Z}_{2} \times \mathbb{Z}_{2} \times \mathbb{Z}_{2} \times \mathbb{Z}_{3}\right)\right)=2$.

For the ring $R=\mathbb{Z}_{2} \times \mathbb{Z}_{2} \times \mathbb{Z}_{8}$, the zero-divisor graph $\Gamma(R)$ contains $K_{3,7}$ with bipartite sets $X=\{(1,0,0)$,

The original article can be found online at https://doi.org/10.1007/ s00500-019-04345-0.

T. Asir

asirjacob75@gmail.com

K. Mano

manokavi5@gmail.com

T. Tamizh Chelvam

tamche59@gmail.com

1 Department of Mathematics-DDE, Madurai Kamaraj University, Madurai, Tamil Nadu 625 021, India

2 Department of Mathematics, Manonmaniam Sundaranar University, Tirunelveli, Tamil Nadu 627 012, India

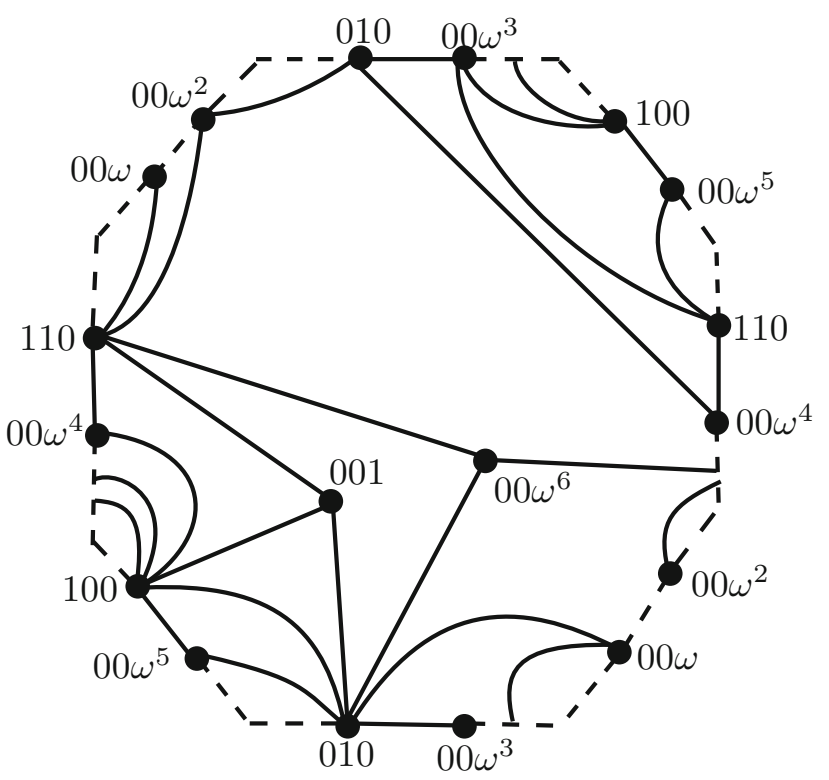

Fig. 1 An embedding of $\Gamma\left(\mathbb{Z}_{2} \widetilde{\mathbb{Z}_{2}} \times \mathbb{F}_{8}\right)$ in $S_{2}$

$(1,1,0),(0,1,0)\}$ and $Y=\{(0,0,1),(0,0,2),(0,0,3)$, $(0,0,4),(0,0,5),(0,0,6),(0,0,7)\}$. Note that $\gamma\left(K_{3,7}\right)=$ 2 and there are 6 rectangular faces and 3 hexagonal faces in any $S_{2}$-embedding of $K_{3,7}$. Now, the vertices $(0,1,4)$ and $(1,0,4)$ would have to be embedded in a face of $K_{3,7}$. Since $((0,1,4),(1,0,4)) \in E(\Gamma(R))$, we observe that they are attached into a single face of $S_{2}$ embedding of $K_{3,7}$. Further, in $\Gamma(R)$, the neighbor of $(0,1,4)$ contains the vertices $(0,0,2),(0,0,4),(0,0,6)$, $(1,0,0)$ and the neighbor of $(1,0,4)$ contains the vertices $(0,0,2),(0,0,4),(0,0,6),(0,1,0)$. But none of the hexagonal face adopt such 9 edges together. Therefore $\gamma\left(\Gamma\left(\mathbb{Z}_{2} \times\right.\right.$ $\left.\left.\mathbb{Z}_{2} \times \mathbb{Z}_{8}\right)\right) \geq 3$ and thus $\gamma\left(\Gamma\left(\mathbb{Z}_{2} \times \mathbb{Z}_{2} \times \mathbb{Z}_{2}[x] /\left(x^{3}\right)\right)\right) \geq 3$.

From the above observation, we have to remove the two rings $\mathbb{Z}_{2} \times \mathbb{Z}_{2} \times \mathbb{Z}_{8}$ and $\mathbb{Z}_{2} \times \mathbb{Z}_{2} \times \mathbb{Z}_{2}[x] /\left(x^{3}\right)$ from the list given in Theorem 4 [1]. Hence the revised statement of Theorem 4 [1] is as follows: 


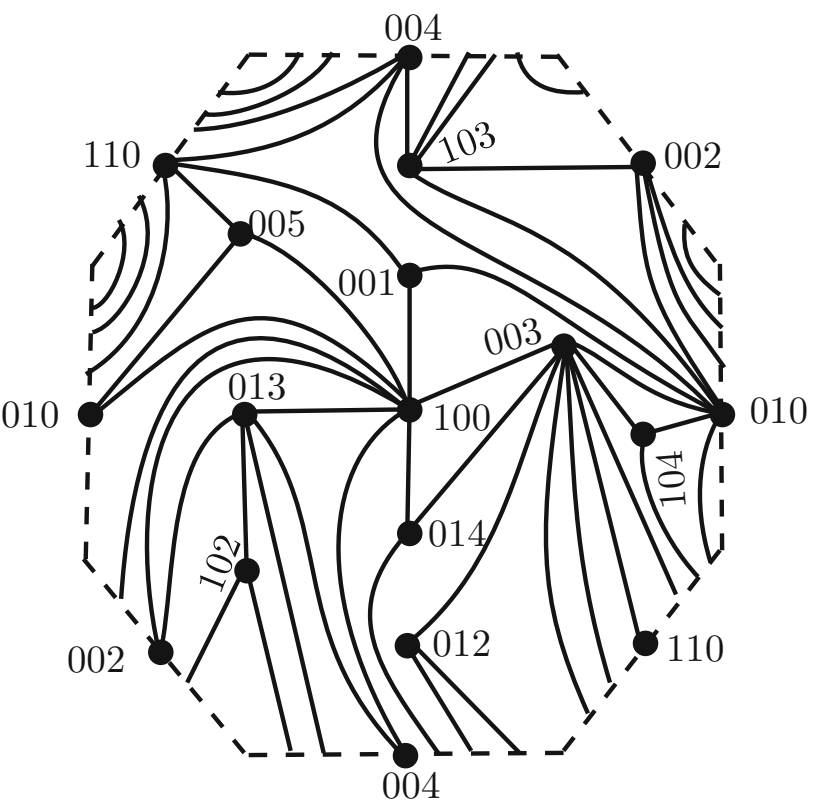

Fig. 2 The embedding of $\Gamma\left(\mathbb{Z}_{2} \widetilde{\times \mathbb{Z}_{2}} \times \mathbb{Z}_{6}\right)$ in $S_{2}$

Theorem 1 Let $R$ be a non-local commutative ring. Then the genus of the zero-divisor graph is 2 if and only if $R$ is isomorphic to one of the following 31 rings:

$$
\begin{aligned}
& \mathbb{F}_{4} \times \mathbb{F}_{8}, \mathbb{F}_{4} \times \mathbb{F}_{9}, \mathbb{F}_{4} \times \mathbb{F}_{11}, \mathbb{Z}_{5} \times \mathbb{Z}_{7}, \mathbb{Z}_{4} \times \mathbb{Z}_{8}, \\
& \mathbb{Z}_{2}[x] /\left(x^{2}\right) \times \mathbb{Z}_{8}, \mathbb{Z}_{4} \times \mathbb{Z}_{2}[x] /\left(x^{3}\right), \\
& \mathbb{Z}_{2}[x] /\left(x^{2}\right) \times \mathbb{Z}_{2}[x] /\left(x^{3}\right), \mathbb{Z}_{2}[x] /\left(x^{2}\right) \times \mathbb{Z}_{9}, \\
& \mathbb{Z}_{4} \times \mathbb{Z}_{3}[x] /\left(x^{2}\right), \mathbb{Z}_{2}[x] /\left(x^{2}\right) \times \mathbb{Z}_{3}[x] /\left(x^{2}\right), \\
& \mathbb{Z}_{4} \times \mathbb{Z}_{9}, \mathbb{Z}_{2} \times \mathbb{Z}_{25}, \mathbb{Z}_{2} \times \mathbb{Z}_{5}[x] /\left(x^{2}\right), \mathbb{Z}_{2} \times \mathbb{Z}_{16}, \\
& \mathbb{Z}_{2} \times \mathbb{Z}_{2}[x] /\left(x^{4}\right), \mathbb{Z}_{2} \times \mathbb{Z}_{4}[x] /\left(x^{2}-2, x^{4}\right), \\
& \mathbb{Z}_{2} \times \mathbb{Z}_{4}[x] /\left(x^{3}-2, x^{4}\right), \mathbb{Z}_{2} \times \mathbb{Z}_{4}[x] /\left(x^{3}+x^{2}-2, x^{4}\right), \\
& \mathbb{F}_{4} \times \mathbb{Z}_{8}, \mathbb{F}_{4} \times \mathbb{Z}_{2}[x] /\left(x^{3}\right), \mathbb{F}_{4} \times \mathbb{Z}_{9}, \mathbb{F}_{4} \times \mathbb{Z}_{3}[x] /\left(x^{2}\right), \\
& \mathbb{Z}_{2} \times \mathbb{Z}_{2} \times \mathbb{F}_{8}, \mathbb{Z}_{2} \times \mathbb{Z}_{2} \times \mathbb{F}_{9}, \mathbb{Z}_{2} \times \mathbb{Z}_{2} \times \mathbb{Z}_{11}, \\
& \mathbb{Z}_{2} \times \mathbb{Z}_{3} \times \mathbb{Z}_{4}, \mathbb{Z}_{2} \times \mathbb{Z}_{3} \times \mathbb{Z}_{2}[x] /\left(x^{2}\right), \mathbb{Z}_{2} \times \mathbb{Z}_{3} \times \mathbb{Z}_{5}, \\
& \mathbb{Z}_{3} \times \mathbb{Z}_{3} \times \mathbb{F}_{4}, \mathbb{Z}_{2} \times \mathbb{Z}_{2} \times \mathbb{Z}_{2} \times \mathbb{Z}_{3} .
\end{aligned}
$$

Moreover, in the diagram of the $S_{2}$-embedding of $\Gamma \widetilde{\left(\mathbb{Z}_{2} \times\right.}$ $\left.\mathbb{Z}_{3} \times \mathbb{Z}_{4}\right)$ provided in Fig. 7 [1], the vertex $(2,0,2)$ was misprinted. There is no such vertex in $\Gamma\left(\mathbb{Z}_{2} \times \mathbb{Z}_{3} \times \mathbb{Z}_{4}\right)$ and hence wrongly included vertex $(2,0,2)$ is deleted from the $S_{2}$-embedding of $\Gamma\left(\mathbb{Z}_{2} \times \widetilde{\mathbb{Z}_{3}} \times \mathbb{Z}_{4}\right)$ given in Fig. 7 [1].
Acknowledgements The authors would like to thank G. Kalai Murugan and P. Vignesh, Department of Mathematics, Thiruvalluvar University, Vellore, India for pointing out the errors. The research of T. Asir was in part supported by a grant from The Science and Engineering Research Board (SERB-MATRICS Project - Ref. MTR/2017/000830). The research of K. Mano was in part supported by a fellowship from The University Grants Commission (Rajiv Gandhi National Fellowship - F1-17.1/2014-15/RGNF-2014-15-SC-TAM-85000). The research of T. Tamizh Chelvam is supported by CSIR Emeritus Scientist Scheme of Council of Scientific and Industrial Research (No.21 (1123)/20/EMRII).

\section{Compliance with ethical standards}

Conflict of interest The authors declare that there is no conflict of interests regarding the publishing of this paper.

Ethical approval This article does not contain any studies with human participants or animals performed by any of the authors.

\section{Reference}

1. Asir T, Mano K (2020) Classification of non-local rings with genus two zero-divisor graphs. Soft Comput 24:237-245

Publisher's Note Springer Nature remains neutral with regard to jurisdictional claims in published maps and institutional affiliations. 